\title{
The role of ultrasound elastography and virtual touch tissue imaging in the personalized prediction of lymph node metastasis of breast cancer
}

\author{
Jue Wang ${ }^{1}$, Zhifei Ben ${ }^{1}$, Shanshan Gao ${ }^{1}$, Shuyi Lyu ${ }^{2}$, Xiuzhi Wei ${ }^{1}$ \\ ${ }^{1}$ Department of Ultrasound, Hwa Mei Hospital, University of Chinese Academy of Sciences, Ningbo Institute of Life and Health Industry, \\ University of Chinese Academy of Sciences, Ningbo, China; ${ }^{2}$ Department of Interventional Therapy, Hwa Mei Hospital, University of Chinese \\ Academy of Sciences, Ningbo Institute of Life and Health Industry, University of Chinese Academy of Sciences, Ningbo, China \\ Contributions: (I) Conception and design: All authors; (II) Administrative support: All authors; (III) Provision of study materials or patients: All \\ authors; (IV) Collection and assembly of data: All authors; (V) Data analysis and interpretation: All authors; (VI) Manuscript writing: All authors; (VII) \\ Final approval of manuscript: All authors. \\ Correspondence to: Xiuzhi Wei. Department of Ultrasound, Hwa Mei Hospital, University of Chinese Academy of Sciences, Ningbo Institute of Life \\ and Health Industry, University of Chinese Academy of Sciences, No. 41 Xibei Road, Ningbo, China. Email: wxz09030830@163.com.
}

Background: This study examined the effects of different ultrasound imaging technologies in the identification and prediction of axillary lymph node metastasis of breast cancer. It also investigated the relationship between human papilloma virus (HPV) infection and axillary lymph node metastasis.

Methods: Eighty-five female patients diagnosed with breast masses participated in this study. Each patient underwent a conventional ultrasound, ultrasonic elastography, and virtual touch tissue imaging quantification (VTIQ). The differential diagnosis efficiency of a conventional ultrasound, ultrasound elastography, VTIQ, and ultrasound elastography combined with VTIQ technology was compared with a pathological diagnosis, which represents the gold standard. 85 axillary lymph node tissues and 25 normal breast tissues were used to detect HPV positive infection rate differences in different tissues.

Results: The results showed that metastatic lymph nodes and reactive lymph node hyperplasia accounted for $54.12 \%$ and $45.88 \%$ of the 85 axillary lymph nodes of breast cancer, respectively. The conventional ultrasound, ultrasound elastography, and VTIQ scores of metastatic lymph nodes were significantly higher than those of reactive lymph node hyperplasia $(\mathrm{P}<0.05)$. The diagnostic sensitivity $(\mathrm{Se})(91.30 \%)$, specificity (Sp) (92.31\%), accuracy (Ac) (91.76\%), positive predictive value (PPV) (93.33\%), and negative predictive value (NPV) (90.00\%) of ultrasound elastography combined with VTIQ technology were the highest among the diagnostic efficiency test results of different computer ultrasound imaging technologies. The positive infection rate of HPV in metastatic lymph node tissues was significantly higher than that in reactive lymph node hyperplasia and normal breast tissues $(\mathrm{P}<0.05)$.

Conclusions: Combining ultrasound elastography with VTIQ technology has high value in the differential diagnosis of axillary lymph nodes of breast cancer. Further, it appears that HPV infection may have an etiological role in lymph node metastasis in breast cancer patients.

Keywords: Axillary lymph node metastasis in breast cancer; human papilloma virus infection (HPV infection); computer ultrasonic imaging technology; metastatic lymph nodes; reactive lymph node hyperplasia

Submitted Feb 03, 2021. Accepted for publication Apr 20, 2021.

doi: $10.21037 /$ gs-21-199

View this article at: http://dx.doi.org/10.21037/gs-21-199 


\section{Introduction}

Breast cancer is a common female malignant tumor. In recent years, breast cancer has become more and more common among younger women. Breast cancer has gradually become the 6th leading cause of death among female malignant tumors. Lymph node metastasis is one of the main and common ways of metastasis of breast cancer, especially axillary lymph node metastasis of breast cancer is the most important and common part of lymph node metastasis of breast cancer. Once III-IV breast cancer cells invade the lymphatic vessels of the breast, on the one hand, they can stay in the lymphatic vessels and continue to grow and reproduce, causing obstruction of the lymphatic vessels and causing lymphatic reflux, resulting in spread in the breast and skin edema; on the other hand, the cancer cells will sooner or later transfer to the regional lymph nodes in the form of emboli, resulting in lymphatic spread. Lymph node metastasis is not only the most important feature to determine the prognosis of breast cancer, but also one of the important factors to evaluate the prognosis of breast cancer. The presence of metastasis in the axillary lymph node affects the prognosis and treatment decisions of breast cancer patients (1). In clinical practice, axillary lymph node dissection is often used to treat breast cancer. However, this kind of operation causes harm to patients and can give rise to various complications. Thus, it is essential that the status of axillary lymph nodes in breast cancer patients is effectively identified to avoid the harm that results from the surgical treatment, and improve the prognosis of breast cancer patients.

At present, a variety of computer imaging technologies have been used to identify lymph nodes, including Computer tomography (2). A conventional ultrasound is the preferred computer imaging technique for the clinical evaluation of axillary lymph node differentiation in breast cancer. The emergence of elastography technology has greatly compensated for the shortcomings of misdiagnoses and the high rate of missed diagnoses in conventional ultrasound technology (3). In recent years, virtual touch tissue imaging quantification (VTIQ) technology has become a popular research area in the field of computer ultrasound imaging, as it can accurately locate the region of interest and be used to undertake quantitative and qualitative analyses of lesions. VTIQ technology has been widely used in the diagnosis of liver and other organ diseases $(4,5)$. To enhance treatment effects and improve the prognosis and quality of life of breast cancer patients, the use of computer imaging technology to identify the nature of axillary lymph node metastasis of breast cancer patients has great importance.

Human papilloma virus (HPV) can cause benign and malignant proliferative diseases, such as cervical cancer, in women by infecting the body's epithelial tissue. Numerous studies have shown that HPV infection is closely related to the occurrence and development of breast cancer (6). However, the association between HPV infection and axillary lymph node metastasis in breast cancer has not yet been proven. In the present study, female patients with breast masses were examined to explore the efficiency differences of different computer imaging technologies in the differential diagnosis of axillary lymph node properties of breast cancer. The relationship between HPV infection and the axillary lymph node nature of breast cancer was also investigated. The results of this study should improve the differentiation efficiency of axillary lymph node in breast cancer and provide a theoretical basis for other experts to explore the relationship between HPV infection and axillary lymph node metastasis. We present the following article in accordance with the STARD reporting checklist (available at http://dx.doi.org/10.21037/gs-21-199).

\section{Methods}

\section{Research objects}

Eighty-five patients with breast masses, who were admitted to hospital from June 2018 to June 2019, participated in the study. All of the patients were female, and aged from 35 to 78 (with an average age of $42.36 \pm 8.67$ ). All of the patients were single cases. The diameters of the masses ranged from $0.45-4.54 \mathrm{~cm}$ (with an average of $2.88 \pm 0.72 \mathrm{~cm}$ ).

To be eligible to participate in this study, patients had to meet the following inclusion criteria: (I) have not undergone radiotherapy or chemotherapy at the time of the breast ultrasound examination; (II) have not undergone a breast biopsy before the breast ultrasound examination; (III) not be pregnant or lactating; and (IV) have complete clinical data and clear collected images, consistent with the requirements of the test. Patients were excluded from the study, if they met the following exclusion criteria: (I) had received a prosthesis implantation; (II) had undergone a breast abscess incision and drainage before diagnosis and treatment; (III) had a breast mass rupture or pus flow; and/or (IV) more than 30 days had elapsed between the ultrasonic diagnosis and the surgical treatment of the breast. All procedures 
performed in this study involving human participants were in accordance with the Declaration of Helsinki (as revised in 2013). The experimental procedure was approved by the ethics committee of the Hwa Mei Hospital of University of Chinese Academy of Sciences (IRB No. 2018-023), and all patients signed informed consent forms.

\section{Diagnosis and evaluation method based on computer imaging technology}

The Siemens Acuson S3000 with a 9L4 linear array probe (frequency 4-9 $\mathrm{MHz}$ ) equipped with ultrasound elastography and VTIQ technology was used to undertake a conventional ultrasound, ultrasound elastography, and VTIQ examination of each of the patients. The patients assumed a supine or lateral position during the routine ultrasound examination of the breast and axillary lymph nodes. Index information of lymph node blood typing, blood flow classification, length diameter, and short diameter of the largest section of lymph node, cortical thickness (CT), and so on were collected and detected. The elastography function was turned on to obtain images for evaluation of each indicator. Switching to VTIQ technology, the VTIQ value of the same depth was obtained and the average value was taken.

The blood flow typing of lymph nodes was based on the evaluation method of Yang et al. (2020) (7). Under this method, Type I indicates no for blood (0 points), Type II indicates the gate of the lymphatic type (1 point), Type III indicates a peripheral type ( 2 points), and Type IV indicates a mixed type ( 3 points).

The degree of blood flow richness was determined according to Adler grading (8). Under this grading, Level 0 indicates no obvious blood flow signal in the lymph node $(0$ points), Level 1 indicates that the spot blood flow signal is less than 2 points in the lymph node (1 point), Level 2 indicates that the spot blood flow signal is less than 4 points in the lymph node ( 2 points), and level 3 indicates that the spot blood flow signal is more than 4 points in the lymph node (3 points). Levels $0-1$ indicates a reactive lymph node hyperplasia, while Levels 2-3 indicates a metastatic lymph node.

The conventional ultrasonic evaluation index was conducted in accordance with Jiang et al.'s method (2019) (9). Under this method, the ratio of the longitudinal and lateral axes of lymph nodes was $\geq 2$ ( 1 point), the ratio of the longitudinal and lateral axes of lymph nodes was $<2$ (2 points), the CT was $<3 \mathrm{~mm}$ (1 point), and the CT was $\geq 3 \mathrm{~mm}$ (2 points).
The degree of blood flow richness was determined according to Adler's grading. An ultrasonic assisted elasticity score was $1-2$ (0 points), the elasticity score was 3 (1 point), and the elasticity score was 4 ( 2 points).

Under the VTIQ evaluation index, a mean value of VTIQ $\geq 1.83 \mathrm{~m} / \mathrm{s}$ indicates a metastatic lymph node (2 points), and a VTIQ $<1.83 \mathrm{~m} / \mathrm{s}$ indicates reactive lymph node hyperplasia (1 point).

All of the examinations were conducted by two clinicians who held intermediate or higher titles. The clinicians adopted a double-blind examination method. In cases of disagreement, a meeting was held to discuss the diagnosis.

\section{HPV infection rate analysis}

All of the patients were treated with modified radical mastectomy for primary breast cancer. Breast cancer tissues were taken from the patients, and 25 pieces of normal breast tissues were also taken to serve as a control group. Paraffin sections were then made. After a section was dewaxed, it was placed in a $1.5 \mathrm{~mL}$ sterilized centrifuge tube. After $1 \mathrm{~mL}$ of xylene was added to the mixture, the sections were centrifuged at 12,000 rpm for $2 \mathrm{~min}$. The supernatant was discarded and the step was repeated twice. $1 \mathrm{~mL}$ ethanol was added and mixed well. The supernatant was discarded. The step was repeated twice. After digestion, the deoxyribonucleic acid (DNA) solution was obtained.

The obtained DNA solution was used as a template for HPV-DNA detection. The main steps included polymerase chain reaction amplification, DNA hybridization, and Luminex 200 detection. After mixing the amplified HPVDNA solution with the configured buffer at a ratio of $4: 1$, it was placed in the sample hole containing agarose gel for amplification electrophoresis. If the signal of the HPV specific probe was greater than 150 , the infection was positive, and if it was less than 150 , the infection was negative.

\section{Statistical analysis}

SPSS19.0 software was used for the statistical analysis. The measurement data are expressed as mean \pm standard deviation $(\bar{x} \pm \mathrm{SD})$. An independent sample $\mathrm{t}$ test was used for the analysis. The qualitative data were analyzed using the $\chi^{2}$ test. The differences between the diagnostic sensitivity (Se), specificity (Sp), accuracy (Ac), positive predictive value (PPV), and negative predictive value (NPV) of the different imaging methods were compared with the pathological 
examination results, which represented the gold standard. A $\mathrm{P}<0.05$ was considered statistically significant.

\section{Results}

\section{Patbological diagnosis results}

A total of 85 axillary lymph nodes were obtained from 85 breast cancer patients, and these 85 axillary lymph nodes were pathologically diagnosed. As Figure 1 shows, metastatic lymph nodes accounted for $54.12 \%$ and reactive lymph node hyperplasia accounted for $45.88 \%$ of cases.

\section{Diagnostic results of the conventional ultrasound}

As Figure 2 shows, the lymph node morphology of this

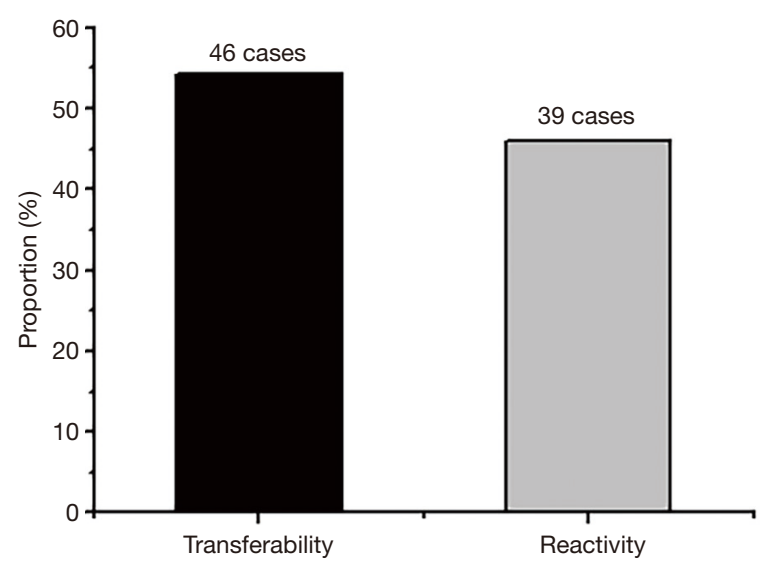

Figure 1 Pathological diagnoses of axillary lymph nodes in breast cancer patients.
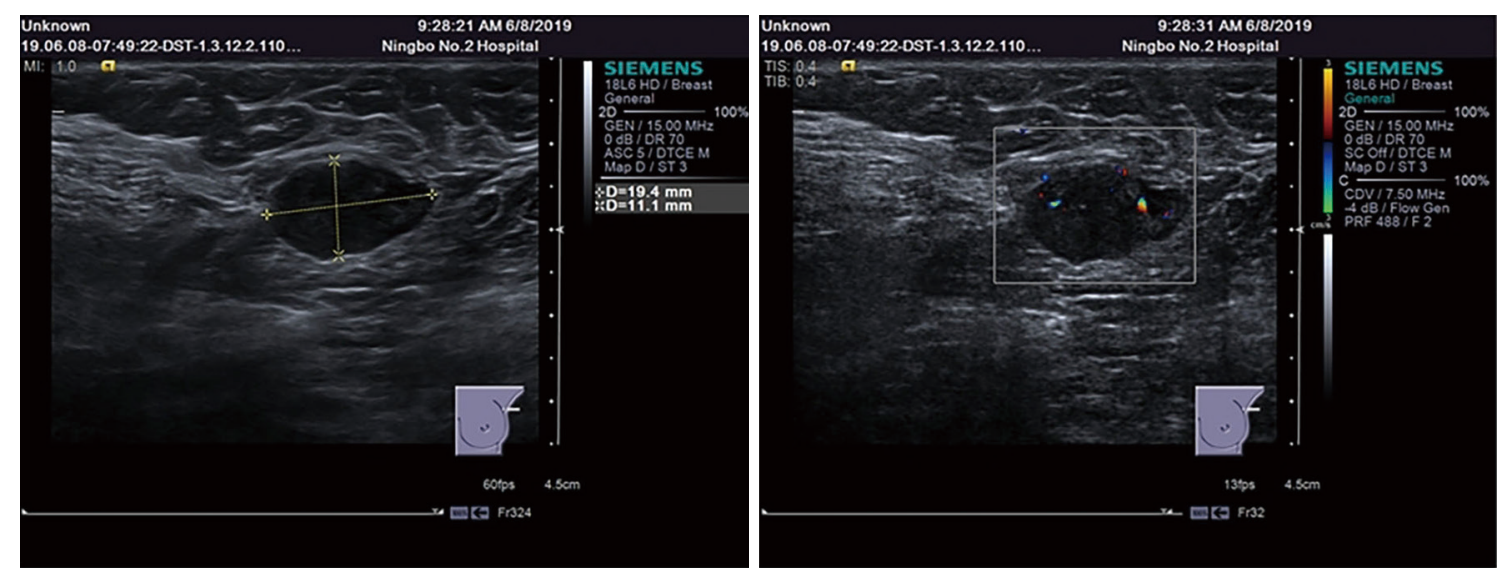

particular patient was irregular, the CT was more than $3 \mathrm{~mm}$, the blood flow signal was Level 3, the blood flow type was mixed, and the conventional ultrasound score was 7 , which indicated that the lymph nodes were malignant. The pathological examination showed metastatic lymph nodes.

As Figure 3 shows, the lymph node morphology of this particular patient was irregular, the CT was less than $3 \mathrm{~mm}$, and there was no obvious blood flow signal. The conventional ultrasound score was 4 points, which indicated that the lymph nodes were benign. A pathological examination showed reactive lymph node hyperplasia.

Subsequently, differences in the conventional ultrasound assessments of reactive and metastatic lymph nodes were compared. As Figure $4 A$ shows, some reactive lymph node hyperplasia was also diagnosed if the aspect ratio of the lymph nodes (L/S) was $\geq 2(28.21 \%)$, the CT was $\geq 3 \mathrm{~mm}$ $(23.08 \%)$, the blood flow grade was a Level 2 or Level 3 (17.95\%), and the blood type was Type III and Type IV (25.64\%). The differences in the conventional ultrasound scores of the metastatic lymph nodes and the reactive lymph node hyperplasia were compared. As Figure $4 B$ shows, the conventional ultrasound score of metastatic lymph nodes was significantly higher than that of reactive lymph node hyperplasia $(\mathrm{P}<0.05)$.

\section{Diagnosis results of the ultrasound elastography}

As Figure 5 shows, the blood flow of the axillary lymph node was Level 3, the elasticity score was 4 points, and the overall score was 8 . The pathological diagnosis revealed an invasive ductal carcinoma with metastatic axillary lymph nodes.

As Figure 6 shows, the blood flow classification of the left

Figure 2 Conventional ultrasound diagnosis of metastatic lymph nodes. 

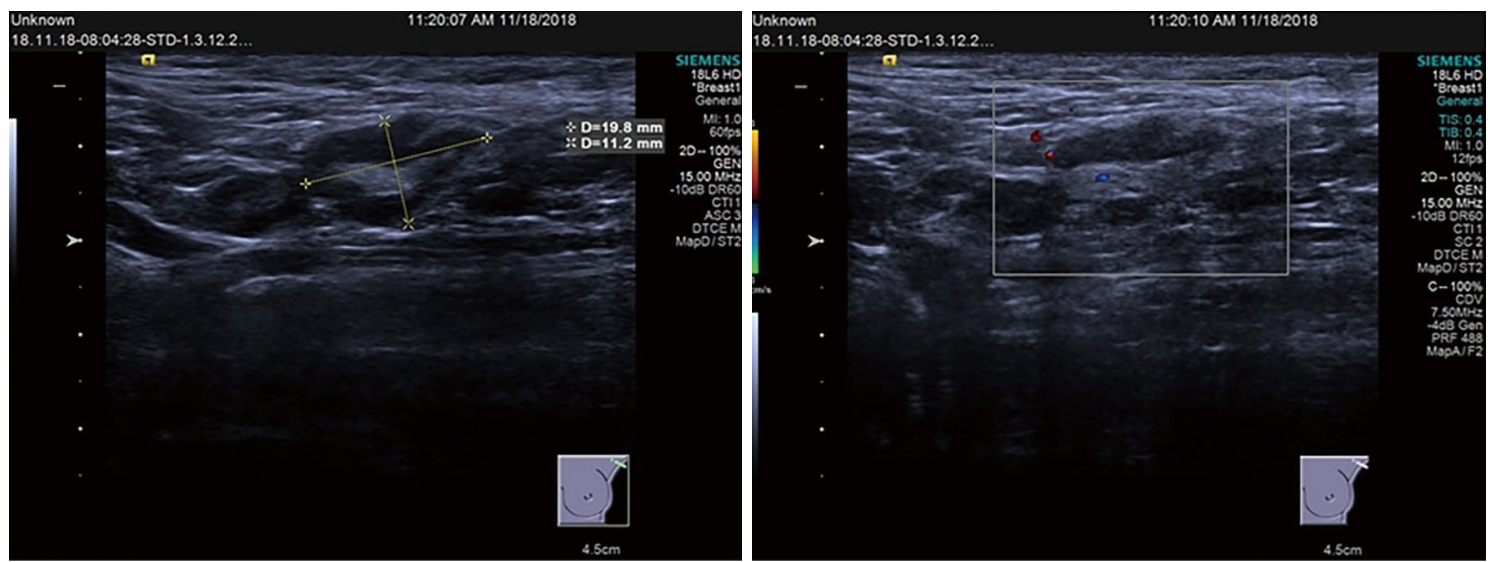

Figure 3 Conventional ultrasound diagnosis of reactive lymph node hyperplasia.
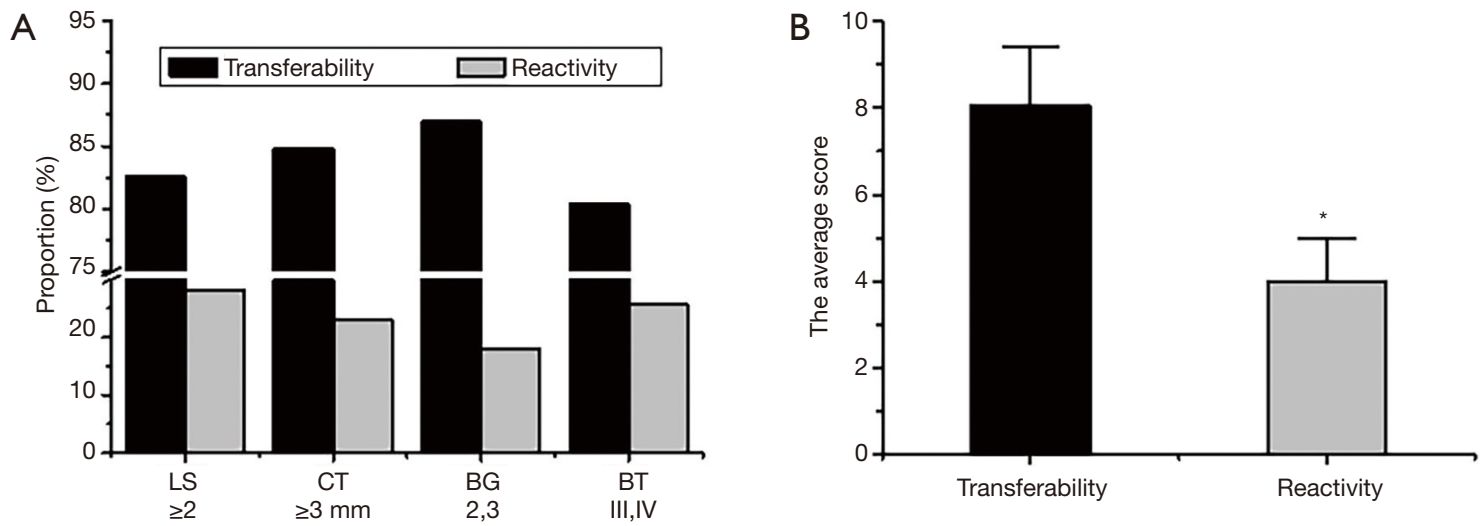

Figure 4 Conventional ultrasound evaluation results of two types of lymph nodes. (A) The differences in diagnostic probability based on different evaluation indicators; (B) differences in conventional ultrasound scores. * indicates that the difference between the two groups was statistically significant $(\mathrm{P}<0.05)$.

axillary lymph node for patients was Grade 1, bleeding type was Type 1 , the elastic score was 2 points, and the overall score was 3 . The pathological diagnosis showed invasive ductal carcinoma with no axillary lymph node metastasis.

Next, evaluation differences of reactive and metastatic lymph nodes by an ultrasound elastography were compared. As Figure 7 shows, the ultrasonic elastography score of the metastatic lymph nodes was significantly higher than that of the reactive lymph node hyperplasia score $(\mathrm{P}<0.05)$.

\section{Diagnosis results of VTIQ imaging}

As Figure $8 \mathrm{~A}$ shows, there was no lymph node metastasis, and the pathological diagnosis showed reactive lymph node hyperplasia. The patient had developed obvious lymph node metastasis, indicating malignancy. Similarly, the pathological diagnosis showed there were metastatic lymph nodes. Figure $8 B$ shows the mean VTIQ of metastatic lymph nodes was $2.55 \pm 0.48$, while the mean VTIQ of reactive lymph node hyperplasia was $1.48 \pm 0.21$. Thus, the mean VTIQ of metastatic lymph nodes was significantly higher than that of reactive lymph node hyperplasia $(\mathrm{P}<0.05)$.

\section{Comparison of the diagnostic efficiency of different computer imaging technologies}

The diagnosis efficiency of different computer imaging technologies (i.e., a conventional ultrasound, an ultrasound elastography, VTIQ, an ultrasound elastography combined with VTIQ technology) in metastatic lymph node and 


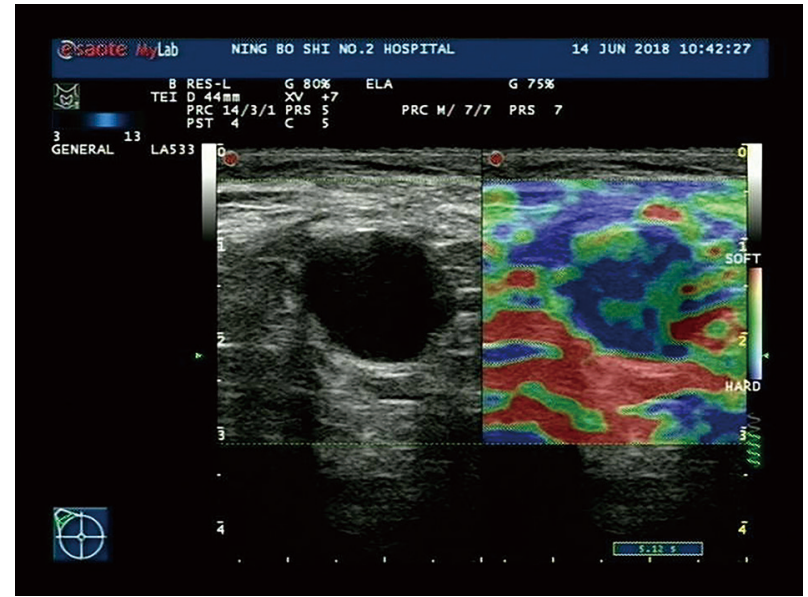

Figure 5 Ultrasound elastography diagnosis of metastatic lymph nodes.

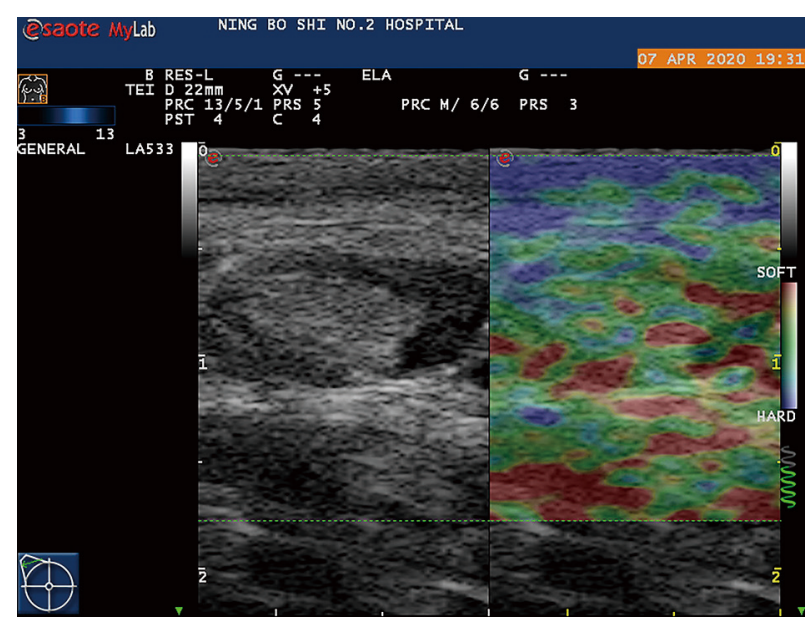

Figure 6 Ultrasound elastography diagnosis of reactive lymph node hyperplasia.

reactive lymph node hyperplasia was compared. The results showed that when different computer imaging technologies were used for the diagnosis of these two types of lymph node metastasis, there were diagnostic errors; however, generally, an ultrasound elastography combined with VTIQ technology had the lowest error diagnosis rate (see Table 1).

The Se (71.74\%), Sp (84.62\%), Ac (77.65\%), PPV (84.62\%), and NPV (71.74\%) were the lowest in conventional ultrasound diagnosis (see Table 2). Conversely, the Se (91.30\%), Sp (92.31\%), Ac (91.76\%), PPV (93.33\%), and NPV $(90.00 \%)$ of ultrasound elastography combined with VTIQ technology were the highest (see Table 2).

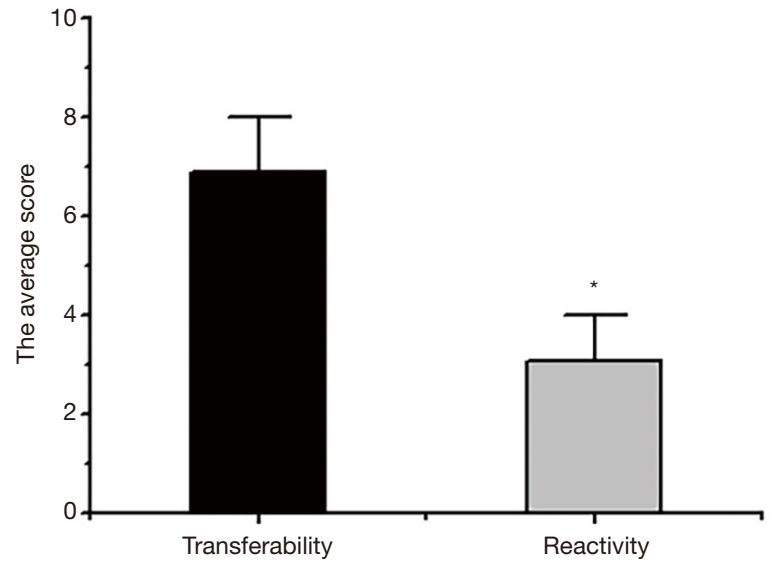

Figure 7 Ultrasound elastography of two types of lymph nodes. * indicates that the difference between the two groups was statistically significant $(\mathrm{P}<0.05)$.

\section{Detection results comparison of HPV infection rate}

As Figure 9 shows, the HPV-positive infection rate of metastatic lymph node tissues was $58.70 \%$, that of reactive lymph node hyperplasia tissues was $15.38 \%$, and that of normal tissues was $4.00 \%$. After comparing the differences, it was found that the HPV positive infection rate of reactive lymph node hyperplasia and normal tissues was significantly lower than that of metastatic lymph node tissues $(\mathrm{P}<0.05)$, and the negative infection rate was significantly higher than that of metastatic lymph node tissues $(\mathrm{P}<0.05)$. The HPV positive infection rate in normal tissues was significantly lower than that in reactive lymph node hyperplasia tissues $(\mathrm{P}<0.05)$, and the negative infection rate was significantly higher than that in reactive lymph node hyperplasia tissues $(\mathrm{P}<0.05)$.

\section{Discussion}

Axillary lymph node metastasis is the main site of breast cancer metastasis. The diagnosis of lymph node metastasis of breast cancer by ultrasound is based on pathological changes. Normal lymph nodes are similar in shape to small kidneys, and the cortex shows uniform hypo echogenicity, while the medulla shows higher echogenicity (10). However, when axillary lymph node metastasis occurs in breast cancer, the cortex of lymph nodes will show centripetal thickening, and in severe cases, the medulla of lymph nodes will completely disappear (11). With the development of computerized ultrasound imaging, high- 

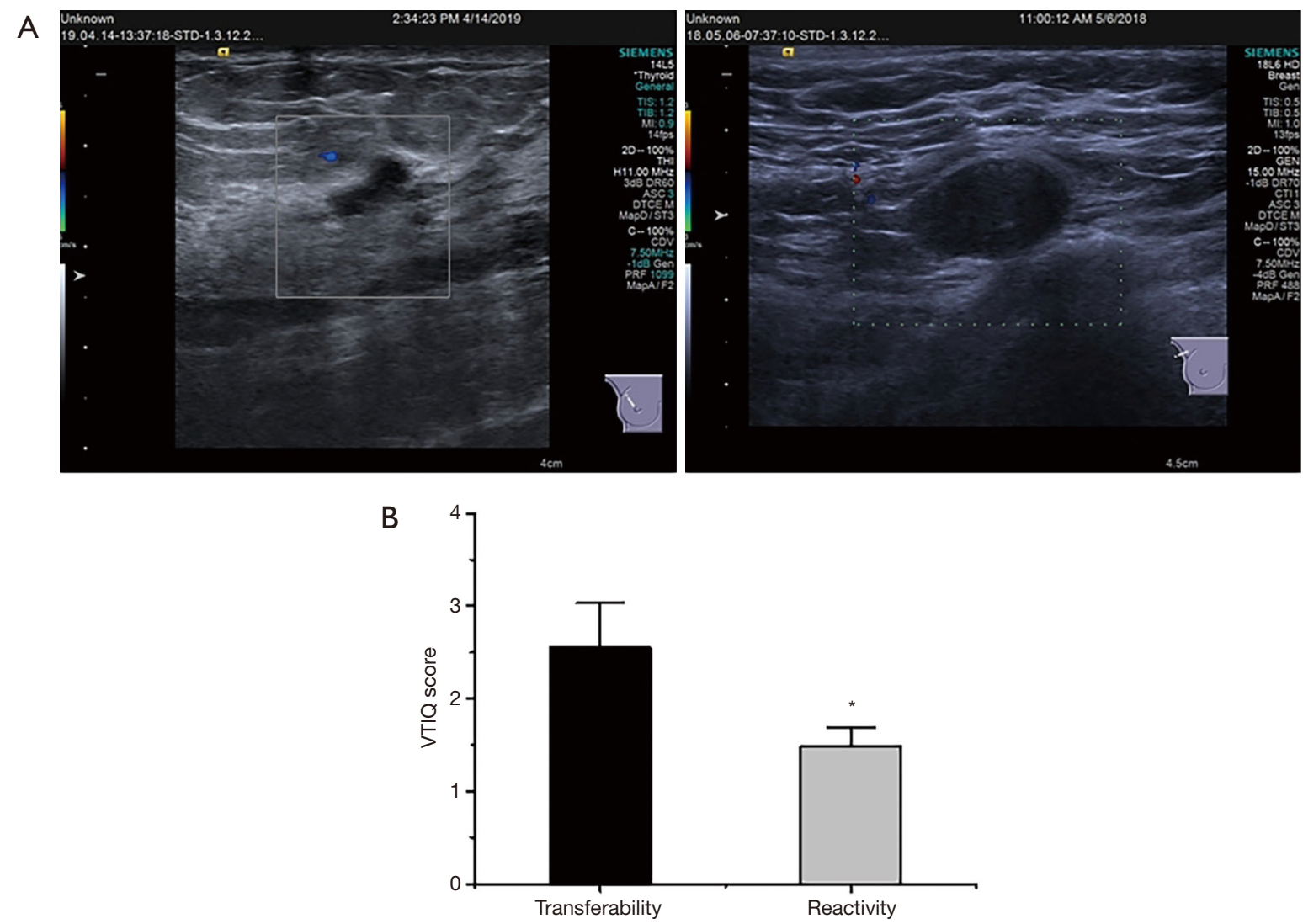

Figure 8 VTIQ evaluation results of two types of lymph nodes. (A) VTIQ evaluation results of two types of lymph nodes; (B) statistical results of VTIQ of two types of lymph nodes. * indicates that the difference between the two groups was statistically significant $(\mathrm{P}<0.05)$. VTIQ, virtual touch tissue imaging quantification.

frequency ultrasounds have been used to detect superficial lymph nodes. However, the differential diagnosis Se of conventional two-dimensional ultrasound technology in axillary lymph node metastasis of breast cancer is not ideal. In this study, the diagnostic Se and Sp of this technique were $71.74 \%$ and $84.62 \%$, respectively. These results were similar to those of Coronado-Gutiérrez et al. (2019) (12). Computer elastography can accurately reflect the hardness of the tissue, so as to semi-quantitatively identify the type of axillary lymph node metastasis of breast cancer. The present study showed that the Se and Sp of ultrasound elastography were $80.43 \%$ and $89.74 \%$ respectively in the differential diagnosis of axillary lymph node metastasis of breast cancer. These results were similar to those of Luo et al. (2019) (13).

At present, ultrasound is one of the main means to diagnose breast cancer and distinguish benign from malignant foci. It has the advantages of low price, noninvasive to the body, simple operation and so on. With the continuous maturity of ultrasonic technology, two kinds of ultrasonic techniques, color Doppler ultrasound and ultrasonic elastography, have emerged successively. At present, color Doppler ultrasound is widely used, mainly by observing the boundary, recovery, shape, blood flow and other conditions of the tumor to judge the nature of the tumor. However, some studies have found that the accuracy of color Doppler ultrasound examination is affected by the size of the focus, patients' own blood flow and other factors, so there will be some missed diagnosis or misdiagnosis. Ultrasonic elastography is a new ultrasonic technology, and its working principle is completely different from that of color Doppler ultrasound. It mainly judges the nature of the focus by detecting the elasticity of the lesion tissue, and is not affected by the blood flow of the lesion size. Ultrasonic elastography is superior to color Doppler ultrasound in missed diagnosis rate, accuracy sensitivity, negative predictive value and so on. It is worthy of clinical application.

In recent years, VTIQ technology has become a 
Table 1 Comparison of diagnostic results of different computer imaging technologies

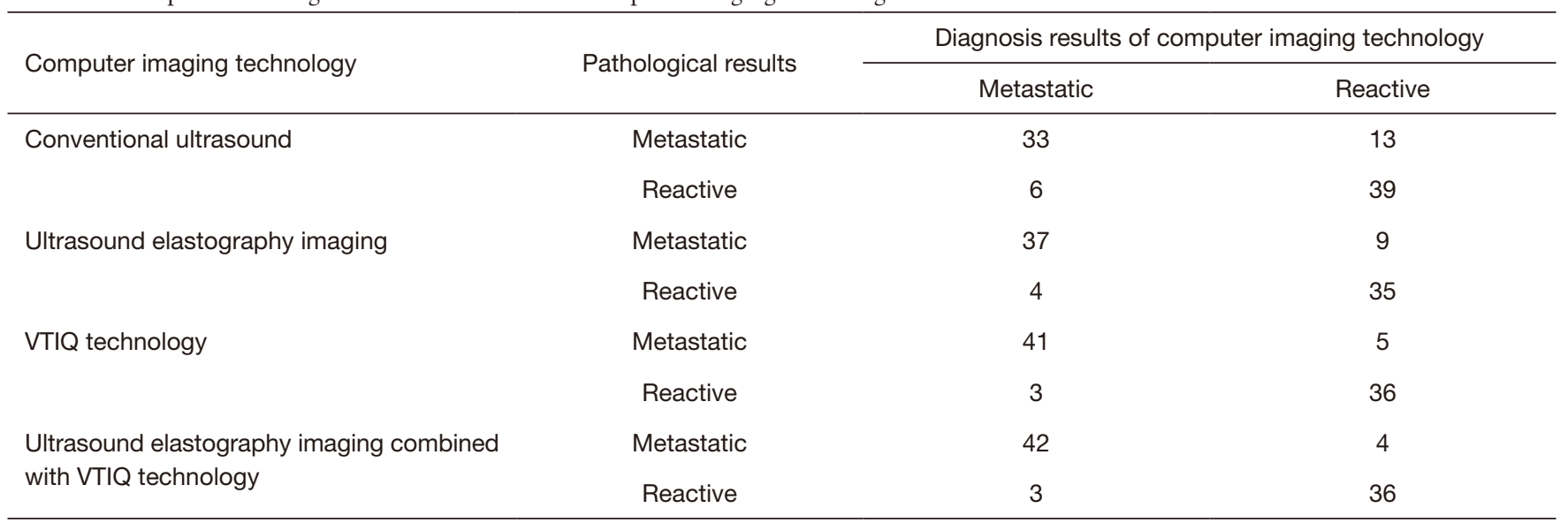

VTIQ, virtual touch tissue imaging quantification.

Table 2 Comparison of diagnostic efficiency of different computer imaging technologies

\begin{tabular}{|c|c|c|c|c|c|}
\hline Computer imaging technology & $\mathrm{Se}(\%)$ & $\mathrm{Sp}(\%)$ & Ac $(\%)$ & PPV (\%) & NPV (\%) \\
\hline Ultrasound elastography imaging & 80.43 & 89.74 & 84.71 & 90.24 & 79.55 \\
\hline VTIQ technology & 89.13 & 92.31 & 90.59 & 93.18 & 87.80 \\
\hline $\begin{array}{l}\text { Ultrasound elastography imaging combined with VTIQ } \\
\text { technology }\end{array}$ & 91.30 & 92.31 & 91.76 & 93.33 & 90.00 \\
\hline
\end{tabular}

Se, sensitivity; Sp, specificity; Ac, accuracy; PPV, positive predictive value; NPV, negative predictive value; VTIQ, virtual touch tissue imaging quantification.

popular research area in computer imaging technology in the medical field. In this study, VTIQ technology was used to identify axillary lymph node metastasis of breast cancer. The results showed that the diagnostic Se and Sp of this technique were $89.13 \%$ and $92.31 \%$, respectively. These percentages were both higher than those found for conventional ultrasound imaging and ultrasound elastography. Thus, consistent with the research results of Liu et al. (2019) (14), these results showed that VTIQ technology has greater value in the differential diagnosis of axillary lymph nodes of breast cancer. VTIQ is a new type of elastic acoustic imaging technology of acoustic pulse radiation force. its principle is that the probe is placed on the body surface without external force, the tissue deformation is driven by the focused pulse wave emitted by the probe, and the probe receives the deformation information. The ultrasonic instrument displays the tissue hardness in four modes: time, displacement, mass and speed, the quality mode is expressed by different color levels, and when the quality is good, it is changed to the speed mode that quantitatively represents the tissue hardness. It makes the quantitative measurement of tissue hardness more reliable. The hardness information of small lesions can be measured. There are some differences in the hardness of different tissues in the mammary gland. the hardness of the nodules is related to their internal composition and arrangement. benign nodules such as adenosis and fibroadenoma contain more soft mucopolysaccharide components, and the arrangement of fibrous stroma and glands is relatively loose. however, malignant nodules such as infiltrating ductal carcinoma contain more dense elastic fibers and stroma, and the stroma can be organized and calcified. And the fibrous interstitial collagenization of most micro-breast cancer will cause a certain pulling effect on the surrounding tissue, and the hardness is significantly higher than that of the surrounding normal tissue, which are the pathological basis for VTIQ to evaluate benign and malignant nodules. VTIQ reflects the quantitative value of 


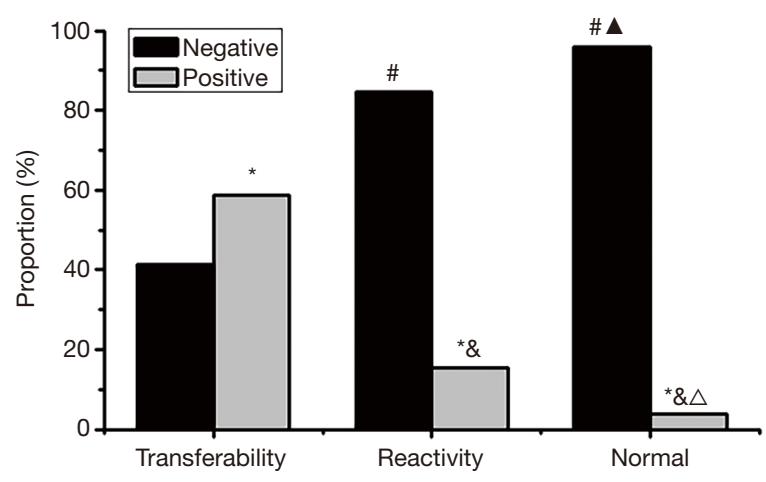

Figure 9 Comparison of HPV infection rates in different types of tissues. * indicates that compared with negative infection rate, the difference was statistically significant $(\mathrm{P}<0.05)$; \# indicates that compared with the negative infection rate of metastatic lymph nodes, the difference was statistically significant $(\mathrm{P}<0.05) ; \&$ indicates that compared with the positive infection rate of metastatic lymph nodes, the difference was statistically significant $(\mathrm{P}<0.05) ; \boldsymbol{\Delta}$ indicates that the difference was statistically significant compared with the negative infection rate of reactive lymph node hyperplasia $(\mathrm{P}<0.05) ; \triangle$ indicates that the difference was statistically significant compared with the positive infection rate of reactive lymph node hyperplasia $(\mathrm{P}<0.05)$. HPV, human papilloma virus.

nodular hardness, and its hardness parameters are obtained by measuring the average value for many times under the condition that the interference factors are fully reduced and the quality mode is well displayed. In addition, the sampling frame is small, and the sampling will not deviate from the nodular range when measuring nodular hardness, so as to ensure the reliability of the measured data. VTIQ has high sensitivity and accuracy.

Ultrasound elastography combined with VTIQ technology was also used in the differential diagnosis of lymph node metastasis in breast cancer. The results showed that the Se and Sp of combined diagnosis were $91.30 \%$ and $92.31 \%$, respectively. The Se and Sp of the combined diagnosis were significantly higher than that of conventional ultrasound and ultrasound elastography, and the Se of diagnosis was significantly higher than that of VTIQ. Thus, the results showed that ultrasound elastography combined with VTIQ appears to effectively improve the diagnostic value of axillary lymph node metastasis in breast cancer.

HPV infection is closely related to breast cancer (15). To explore the relationship between HPV infection and lymph node metastasis of breast cancer, the differences between the infection rates of the HPV virus in metastatic lymph node tissue, reactive lymph node hyperplasia, and normal breast tissue were examined. The results showed that the HPVpositive infection rate in metastatic lymph node tissues was $58.70 \%$, which was significantly higher than that in reactive lymph node hyperplasia tissues, which was $15.38 \%(\mathrm{P}<0.05)$. Thus, HPV infection may play an etiological role in the axillary lymph node metastasis in breast cancer patients.

\section{Conclusions}

In this study, the effectiveness of different computer imaging technologies in the identification of axillary lymph node properties in breast cancer patients was explored. The relationship between HPV infection and axillary lymph node metastasis was analyzed. The results showed that ultrasound imaging technology combined with VTIQ imaging technology had the highest value in the identification of axillary lymph nodes in breast cancer. Further, HPV infection was found to be closely related to the axillary lymph node metastasis of breast cancer. However, the relationship between HPV infection and axillary lymph node metastasis of breast cancer was only preliminarily explored. Subsequently, the sample size should be increased to explore the relationship between HPV infection and axillary lymph node metastasis of breast cancer from the molecular perspective. In summary, the results of this study could be used to greatly improve the surgical treatment effects and the prognosis of breast cancer patients.

\section{Acknowledgments}

Funding: This work was Supported by the Medical Scientific Research Foundation of Zhejiang Province, China (Grant No. 2020KY852, Grant No. 2020KY837, and Grant No. 2019KY596).

\section{Footnote}

Reporting Checklist: The authors have completed the STARD reporting checklist. Available at http://dx.doi.org/10.21037/ gs-21-199

Data Sharing Statement: Available at http://dx.doi. org/10.21037/gs-21-199

Conflicts of Interest: All authors have completed the ICMJE uniform disclosure form (available at http://dx.doi. org/10.21037/gs-21-199). The authors have no conflicts of 
interest to declare.

Ethical Statement: The authors are accountable for all aspects of the work in ensuring that questions related to the accuracy or integrity of any part of the work are appropriately investigated and resolved. All procedures performed in this study involving human participants were in accordance with the Declaration of Helsinki (as revised in 2013). The experimental procedure was approved by the ethics committee of the Hwa Mei Hospital of University of Chinese Academy of Sciences (IRB No. 2018-023), and all patients signed informed consent forms.

Open Access Statement: This is an Open Access article distributed in accordance with the Creative Commons Attribution-NonCommercial-NoDerivs 4.0 International License (CC BY-NC-ND 4.0), which permits the noncommercial replication and distribution of the article with the strict proviso that no changes or edits are made and the original work is properly cited (including links to both the formal publication through the relevant DOI and the license). See: https://creativecommons.org/licenses/by-nc-nd/4.0/.

\section{References}

1. Haffty BG, McCall LM, Ballman KV, et al. Impact of Radiation on Locoregional Control in Women with Node-Positive Breast Cancer Treated with Neoadjuvant Chemotherapy and Axillary Lymph Node Dissection: Results from ACOSOG Z1071 Clinical Trial. Int J Radiat Oncol Biol Phys 2019;105:174-82.

2. Kim H, Park W, Kim SS, et al. Outcome of breast-conserving treatment for axillary lymph node metastasis from occult breast cancer with negative breast MRI. Breast 2020;49:63-9.

3. Kane G, Fleming C, Heneghan H, et al. False-negative rate of ultrasound-guided fine-needle aspiration cytology for identifying axillary lymph node metastasis in breast cancer patients. Breast J 2019;25:848-52.

4. Sun JW, Wang XL, Zhao Q, et al. Virtual touch tissue imaging and quantification (VTIQ) in the evaluation of breast lesions: The associated factors leading to misdiagnosis. Eur J Radiol 2019;110:97-104.

5. Erdogan Iyigun Z, Agacayak F, Ilgun AS, et al. The Role of Elastography in Diagnosis and Staging of Breast CancerRelated Lymphedema. Lymphat Res Biol 2019;17:334-9.

6. De Carolis S, Storci G, Ceccarelli C, et al. HPV DNA Associates With Breast Cancer Malignancy and It Is Transferred to Breast Cancer Stromal Cells by
Extracellular Vesicles. Front Oncol 2019;9:860.

7. Yang WT, Chang J, Metreweli C. Patients with breast cancer: differences in color Doppler flow and gray-scale US features of benign and malignant axillary lymph nodes. Radiology 2000;215:568-73.

8. Kong QF, Lv B, Wang B, et al. Association of von Willebrand factor (vWF) expression with lymph node metastasis and hemodynamics in papillary thyroid carcinoma. Eur Rev Med Pharmacol Sci 2020;24:2564-71.

9. Jiang W, Wei HY, Zhang HY, et al. Value of contrastenhanced ultrasound combined with elastography in evaluating cervical lymph node metastasis in papillary thyroid carcinoma. World J Clin Cases 2019;7:49-57.

10. Hayes AJ, Moskovic E, O'Meara K, et al. Prospective cohort study of ultrasound surveillance of regional lymph nodes in patients with intermediate-risk cutaneous melanoma. Br J Surg 2019;106:729-34.

11. Morency D, Dumitra S, Parvez E, et al. Axillary Lymph Node Ultrasound Following Neoadjuvant Chemotherapy in Biopsy-Proven Node-Positive Breast Cancer: Results from the SN FNAC Study. Ann Surg Oncol 2019;26:4337-45.

12. Coronado-Gutiérrez D, Santamaría G, Ganau S, et al. Quantitative Ultrasound Image Analysis of Axillary Lymph Nodes to Diagnose Metastatic Involvement in Breast Cancer. Ultrasound Med Biol 2019;45:2932-41.

13. Luo S, Yao G, Hong Z, et al. Qualitative Classification of Shear Wave Elastography for Differential Diagnosis Between Benign and Metastatic Axillary Lymph Nodes in Breast Cancer. Front Oncol 2019;9:533.

14. Liu H, Wan J, Xu G, et al. Conventional US and 2-D Shear Wave Elastography of Virtual Touch Tissue Imaging Quantification: Correlation with Immunohistochemical Subtypes of Breast Cancer. Ultrasound Med Biol 2019;45:2612-22.

15. Hamad BJ, Saleh MB. Association Between BRCA1/2 Polymorphism and HPV in Breast Cancer Patients in Thi-Qar/South Iraq. University of Thi-Qar Journal 2019;14:14-22.

(English Language Editor: L. Huleatt)

Cite this article as: Wang J, Ben Z, Gao S, Lyu S, Wei X. The role of ultrasound elastography and virtual touch tissue imaging in the personalized prediction of lymph node metastasis of breast cancer. Gland Surg 2021;10(4):1460-1469. doi: 10.21037/gs-21-199 